Research Paper

\title{
Risk Factors for Fungal Prosthetic Joint Infection
}

\author{
Talha Riaz ${ }^{1,3}$, Aaron J. Tande 3 , Lisa L. Steed ${ }^{1}$, Harry A. Demos², Cassandra D. Salgado, Douglas R. \\ Osmon $^{3}$, Camelia E. Marculescu ${ }^{1 凶}$ \\ 1. Division of Infectious Diseases, Medical University of South Carolina, Charleston, South Carolina; \\ 2. Department of Orthopaedics and Rehabilitation, Medical University of Charleston, Charleston, South Carolina; \\ 3. Division of Infectious Diseases, Mayo Clinic, Rochester, Minnesota. \\ $\triangle$ Corresponding author: Camelia Marculescu, MD, MSCR, Division of Infectious Diseases, 12th floor Rutledge Tower, 135 Rutledge Ave MSC 752, Charleston, \\ SC 29425. Email: Marcule@musc.edu. \\ ( ) The author(s). This is an open access article distributed under the terms of the Creative Commons Attribution License (https://creativecommons.org/licenses/by/4.0/). \\ See http://ivyspring.com/terms for full terms and conditions.
}

Received: 2019.09.17; Accepted: 2020.01.30; Published: 2020.03.26

\begin{abstract}
Background: Fungal prosthetic joint infections (PJls) are rare and often associated with poor outcome; however, risk factors are not well described.

Methods: This was a retrospective case control study among all patients with PJls from 2006-2016 at two major academic centers. Each fungal PJI case was matched 1:1 with a bacterial PJI control by joint (hip, knee, shoulder) and year of diagnosis. We compared demographics, comorbidities, and clinical characteristics between cases and controls using chi square/Fisher's exact or Wilcoxon rank sum test. Independent risk factors were identified with multivariable logistic regression.

Results: Forty-one fungal PJls occurred over the study and $61 \%$ were due to Candida albicans. The hip was involved in $51.2 \%$ of cases, followed by the knee (46.3\%). Compared to bacterial PJI, fungal PJI cases were more likely to have received antibiotics within the previous 3 months $(70.7 \%$ vs $34 \%$, $P=.001)$, wound drainage lasting $>5$ days ( $48 \%$ vs $9 \%, P=.0002)$, had a lower median CRP $(2.95 \mathrm{mg} / \mathrm{dl}$ vs $5.99, P=.013)$ and synovial fluid white blood cell count $\left(13,953 \mathrm{cells} / \mathrm{mm}^{3}\right.$ vs $\left.33,198, P=.007\right)$, and a higher proportion of prior two-stage exchanges ( $82.9 \%$ vs $53.6 \%, P=.008)$. After controlling for center, prolonged wound drainage (OR, 7.3; $95 \% \mathrm{Cl}, 2.02-26.95)$ and recent antibiotics (OR, 3.4; $95 \% \mathrm{Cl}, 1.2-9.3)$ were significantly associated with fungal PJI.

Conclusion: In our study, Candida albicans was the most common species in fungal PJls and prolonged wound drainage and recent antibiotics were independent risk factors. These clinical characteristics may help providers anticipate fungal PJI and adjust management strategies.
\end{abstract}

Key words: Candida albicans, case-control study, fungal PJIs

\section{Introduction}

The number of patients living with implanted joint prostheses continues to increase. Many of these patients are older and require revision of the joint prosthesis due to polyethylene wear or aseptic loosening [1]. Just as for other foreign devices, prosthetic joint infection (PJI) is a well-documented adverse outcome. PJI is among the main reasons for failure of joint arthroplasty [2], causing significant morbidity and mortality $[3,4]$. Treatment requires removal of the infected prosthesis and several weeks of intravenous antibiotics, followed by revision surgery, with an estimated cost of more than $\$ 50,000$ for each episode of infection [5]. The most commonly implanted joints are knee and hip prosthesis, followed by shoulder arthroplasties.

While there are consensus guidelines from the Infectious Diseases Society of America with regards to treatment of bacterial infections of prosthetic joints [1], periprosthetic infections due to fungi are rare [6]. They have been historically difficult to treat as well. As the number of patients undergoing joint implantation is increasing worldwide, incidence of fungal PJIs is expected to rise $[7,8]$. 
Among the fungi, both yeasts as well as molds have been reported to cause PJIs. Candida species account for less than $1 \%$ of all PJIs, with mold infections including Aspergillus species being reported even less frequently $[9,10]$.

Following implantation of a prosthetic hip and knee joint, the risk of infection is 0.5 to 1.0 percent for THAs and 0.5 to 2 percent for TKA $[5,11]$. There have been several proposed risk factors for a fungal PJI. Most of these risk factors are identified from small case series and from observational studies. To our knowledge, no well-designed multicenter study to identify the potential risk factors for fungal PJIs has been done. Identifying potential risk factors will allow the treating physician to identify patients at risk of acquiring fungal infection of joint prosthesis. Also, identifying higher risk patients would allow for better preoperative risk assessment and focused efforts at preventing infection. Additionally, fungal PJIs portend a therapeutic challenge due to lack of any established guidelines and have had historically poor outcomes [12-13, 31]. Therefore, we sought to determine such risk factors among patients cared for at two large academic centers.

\section{Methods}

This was a retrospective case-control study performed at two large academic centers, Center A and Center B, between January 2006 and December 2016. Cases (fungal PJIs) and controls (bacterial PJIs) were matched 1:1 ratio based on year of infection and joint location (hip, knee, or shoulder). The study was approved by the institutional review board at each study site. Center A is a large tertiary care hospital and referral center in the Midwestern United States, whereas Center B is a large regional tertiary care hospital in the Southeastern US. Both centers run robust and high volume orthopedic surgery departments. At Center A, PJI database was used to identify cases with fungal PJI. At Center B, all positive reports of all cultures ordered by orthopedic surgeons were extracted from the laboratory information system. By manual review of the culture data, cases and controls were identified.

Fungal PJI was diagnosed if two or more cultures from synovial fluid or cultures obtained from a bone biopsy, or intraoperative tissue cultures yielded the same microorganism. We excluded patients who had a mixed fungal and bacterial PJI.

We identified 41 cases of fungal PJIs (33 cases from Center $\mathrm{A}$ and 8 cases from Center B).

Characteristics of patients with fungal and bacterial PJIs were compared.

Demographic data included patient's age, gender and race. Comorbid conditions such as diabetes mellitus, chronic kidney disease, underlying immunosuppression, malignancy, hemodialysis dependence and exposure to antibiotics lasting longer than 3 days (systemic antibacterial medications) in the last 3 months were abstracted. We recorded clinical presentation and local wound factors such as presence of erythema, sinus tract, drainage, swelling or blister formation. Duration of symptoms was recorded. Lab data including sedimentation rate, C- reactive protein, synovial fluid leukocyte count and renal function were recorded. Finally, prosthesis related factors, such as loosening, and surgical factors that included soft tissue compromise and history of prior surgeries at the index joint including two stage exchange, were abstracted. Timeline of prior surgeries was from date of index arthroplasty.

Rheumatoid arthritis was defined as per the guidelines of the American Rheumatology Society [14]. Diabetes mellitus and obesity were defined as per the national diabetes data group [15], the National Institute of Health (NIH) and the World Health Organization (WHO) [16], respectively. Any form of systemic steroid use lasting longer than two weeks prior to the diagnosis of PJI was recorded. Malignancies that occurred within the past five years (before the diagnosis of PJI) were recorded as well. Chronic renal insufficiency was defined as having a glomerular filtration rate (GFR) of less than 30 $\mathrm{mL} / \mathrm{min} / 1.73 \mathrm{~m}^{2}$. Wound drainage was defined as any drainage occurring longer than five days before the diagnosis of PJI.

\section{Statistical analysis}

We compared categorical variables between fungal and bacterial PJI groups using chi square or Fisher's exact test as appropriate. Continuous variables were compared with the Wilcoxon rank sum test. All tests were two-sided. Type I error ( $\alpha$ ) was set at 0.05 . Risk factors we considered statistically $(P \leq .05)$ and clinically significant were included in a multivariable logistic regression model in a stepwise fashion (SAS 9.4), Cary, NC). The Hosmer-Lemeshow test was performed to assess goodness-of-fit of the final model.

\section{Results}

The median age of the patients in the study was 64.7 years. Twenty-one $(51 \%)$ of the 41 case patients were female and $36(87 \%)$ were white. The hip was involved in $21(51.2 \%)$ cases, followed by the knee $(46.3 \%)$ and shoulder $(2.4 \%)$. The median duration from the time of initial joint arthroplasty and diagnosis of fungal PJI was 515 days (range 17- 5,949). The clinical characteristics are summarized in Table 1. 
Median BMI was $29 \mathrm{~kg} / \mathrm{m}^{2}$ (range 10-106). One patient had a prior limb amputation accounting for a rather low BMI of $10 \mathrm{~kg} / \mathrm{m}^{2}$. Twenty-one (51.2\%) cases had one to three co-morbid conditions which included diabetes mellitus in eight $(9.5 \%)$, chronic renal insufficiency in five (5\%), underlying immunosuppression in 11 (26.8\%), hemodialysis dependence among six (14\%) and receipt of antibiotics within the previous 90 days in $29(70.7 \%)$ cases. While there were no major differences in age or other comorbid conditions, having received antibiotics within the previous 90 days was noted to be statistically significant $(P=.001)$.

In terms of clinical presentation, pain was present in all cases (100\%), followed by swelling in 30 (73.1\%), presence of a sinus tract in $21(51 \%)$, wound drainage in $20(48 \%)$, erythema in $12(29.2 \%)$ and fever in five cases $(12.1 \%)$.

Median CRP was lower among cases (2.95 $\mathrm{mg} / \mathrm{dl}$, range $0.30-23.9)$ versus controls $(5.99 \mathrm{mg} / \mathrm{dl}$, range $0.04-38.6, P=.0132$ ). Similarly, median synovial fluid leukocyte count was 13,953 cells $/ \mathrm{mm}^{3}$ among cases versus 33,198 cells $/ \mathrm{mm}^{3}$ among controls in whom this data was available $(P=.007)$.

We examined surgical factors including surgical site skin infection, loosening of the prosthesis, soft tissue compromise as well as history of prior operation at the index joint and history of prior two stage exchange.

In the univariable analysis, prior two-stage exchange, receipt of antibiotics within the previous 90 days, wound drainage lasting longer than five days, CRP and synovial fluid leukocyte counts were statistically significant.

In the multivariable logistic regression model, after controlling for the center, presence of wound drainage for more than five days (OR, 7.3; 95\% confidence interval [CI], 2.02-26.95) and receipt of antibiotics within the previous 90 days (OR, 3.4; 95\% CI, 1.2-9.3) were factors significantly associated with fungal PJI.

Tables 2 and 3 enlist the microbiological characteristics of the 41 cases and controls. While Candida albicans was the most common fungal pathogen, the most common bacterial pathogen in our study was Staphylococcus epidermidis.

\section{Discussion}

To our knowledge, this is the first case control study aimed to evaluate risk factors for fungal PJIs. Our data suggest that there are two factors significantly associated with an increased odds of fungal PJIs when compared with bacterial PJIs; antimicrobial therapy within three months before the diagnosis of PJI was associated with a 3.4-fold increased odds of having a fungal PJI, and presence of wound drainage lasting longer than five days prior to the diagnosis of PJI was associated with a 7.3-fold increased odds of fungal PJI.

Table 1. Demographics and Clinical Characteristics of Cases and Controls

\begin{tabular}{|c|c|c|c|}
\hline Variable & $\begin{array}{l}\text { Fungal PJI } \\
(\mathrm{n}-41)\end{array}$ & $\begin{array}{l}\text { Bacterial PJI } \\
(n=41)\end{array}$ & $\begin{array}{l}P \\
\text { Value }\end{array}$ \\
\hline \multicolumn{4}{|l|}{ Demographics } \\
\hline Age median years (range) & $64.7(43.9-81.4)$ & $66.7(14.3-89.1)$ & NS \\
\hline Female & $21(51 \%)$ & $20(48 \%)$ & NS \\
\hline Race white & $36(87 \%)$ & $36(87 \%)$ & NS \\
\hline \multicolumn{4}{|l|}{ Prosthesis location } \\
\hline Hip & $21(51 \%)$ & $21(51 \%)$ & \\
\hline Knee & $19(46 \%)$ & $19(46 \%)$ & \\
\hline Shoulder & $1(2.4 \%)$ & $1(2.4 \%)$ & \\
\hline Joint age median days (range) & $515(17-5,949)$ & $1,321(37-7,595)$ & .057 \\
\hline \multicolumn{4}{|l|}{ Host Factors } \\
\hline BMI median (range) & $29(10-106)$ & $29(24-61)$ & .35 \\
\hline \multicolumn{4}{|l|}{ Comorbid conditions } \\
\hline None & $19(46.3 \%)$ & $20(48.7 \%)$ & \\
\hline $1-3$ & $21(51.2 \%)$ & $20(48.7 \%)$ & \\
\hline$>3$ & $1(2.4 \%)$ & $1(2.4 \%)$ & \\
\hline Diabetes mellitus & $8(19.5 \%)$ & $5(12.1)$ & NS \\
\hline Chronic kidney disease & $5(12.1)$ & $7(17 \%)$ & NS \\
\hline Immunosuppression & $11(26.8)$ & $5(12.1)$ & NS \\
\hline Malignancy & $5(12 \%)$ & $1(2.4 \%)$ & NS \\
\hline AKI/HD & $6(14 \%)$ & $2(4.8 \%)$ & NS \\
\hline Prior antibiotics (in last 3 months) & $29(70.7 \%)$ & $14(34 \%)$ & .001 \\
\hline \multicolumn{4}{|c|}{ Clinical presentation and local wound factors } \\
\hline Erythema & $12(29.2)$ & $18(43.9)$ & NS \\
\hline Sinus tract & $21(51 \%)$ & $12(29.2 \%)$ & .07 \\
\hline Pain & $41(100 \%)$ & $39(95 \%)$ & NS \\
\hline Fever & $5(12.1 \%)$ & $8(19.5 \%)$ & NS \\
\hline Swelling/cyst & $30(73.1 \%)$ & $28(68.2 \%)$ & NS \\
\hline Wound drainage ( $>5$ days) & $20(48 \%)$ & $4(9 \%)$ & .0002 \\
\hline $\begin{array}{l}\text { Duration of symptoms median days } \\
\text { (range) }\end{array}$ & $30.0(1-910)$ & $12.0(1-1,213)$ & .08 \\
\hline \multicolumn{4}{|l|}{ Laboratory data } \\
\hline CRP median (range) (mg/dl) ${ }^{a}\left(39^{a} / 40^{b}\right)$ & $2.95(0.30-23.9)$ & $5.99(0.04-38.6)$ & .0132 \\
\hline Sedimentation rate median (range) (39a) & $45.0(6-130)$ & $55.0(0.9-130)$ & NS \\
\hline Creatinine $(\mathrm{mg} / \mathrm{dl})$ & $0.9(0.5-1.7)$ & $1.0(0.5-9.1)$ & NS \\
\hline $\begin{array}{l}\left.\text { Synovial fluid leukocyte (cells } / \mathrm{mm}^{3}\right) \\
\text { median (range) }\left(24^{\mathrm{a}} / 26^{\mathrm{b}}\right)\end{array}$ & $\begin{array}{l}13,953 \\
(157-84,378)\end{array}$ & $\begin{array}{l}33,198 \\
(350-251,049)\end{array}$ & .007 \\
\hline \multicolumn{4}{|l|}{ Surgical factors } \\
\hline Surgical site infection & $4(9 \%)$ & $1(2.4 \%)$ & NS \\
\hline Loosening & $11(26.8 \%)$ & $9(21.9 \%)$ & NS \\
\hline Soft tissue compromise & $7(17 \%)$ & $2(4.8 \%)$ & NS \\
\hline Prior operation at the index joint & $36(87.8 \%)$ & $28(68.2 \%)$ & .06 \\
\hline Prior two stage exchange & $34(82.9 \%)$ & $22(53.6 \%)$ & .008 \\
\hline
\end{tabular}

Abbreviations: NS, not significant; PJI, prosthetic joint infection. a Number of cases for whom risk factor data were available. ${ }^{b}$ Number of controls for whom risk factor data were available.

Table 2. Microbiological Findings of 41 Patients with Fungal PJls (Cases) Seen at Center A and Center B between 2010 and 2016

\begin{tabular}{ll}
\hline Fungal pathogen & Number of patients \\
\hline Candida parapsilosis & 10 \\
Candida albicans & 25 \\
Candida guilliermondii & 1 \\
Candida tropicalis & 1 \\
Coccidiodes immitis & 1 \\
Candida glabrata & 2 \\
Bipolaris spp. & 1 \\
\hline
\end{tabular}

Abbreviation: PJI, prosthetic joint infection. 
Table 3. Microbiological Findings of 41 Patients With Bacterial PJls (Controls) Seen at Center A and Center B Between 2010 and 2016

\begin{tabular}{ll}
\hline Bacterial pathogen & Number of patients \\
\hline Staphylococcus epidermidis & 18 \\
Group B Streptococcus & 2 \\
Methicillin susceptible S aureus & 6 \\
Microbiologically indeterminate & 1 \\
Enterococcus faecalis & 2 \\
Staphylococcus lugdenensis & 2 \\
Serratia marcescens & 1 \\
Cutibacterium acnes & 2 \\
Streptococcus viridans & 1 \\
Streptococcus mitis & 2 \\
Methicillin resistant S aureus & 2 \\
Micrococcus luteus & 1
\end{tabular}

Abbreviation: PJI, prosthetic joint infection.

Brown, T. S. et al. (2018) 'Periprosthetic Joint Infection With Fungal Pathogens', The Journal of arthroplasty, 33(8), pp. 2605-2612.

In our study, consistent with what has been reported in the literature, the most commonly identified fungus was Candida albicans, which was recovered in twenty-five $(60.9 \%)$ cases followed by Candida parapsilosis $(24.4 \%)[13,30]$ (Table 2). It has been reported that Candida albicans makes large and complex biofilms compared to other candida species $[13,17]$, thus contributing to its pathogenicity. Among the patients included in the bacterial cohort, coagulase negative Staphylococcus was recovered in $43.9 \%$, followed by Staphylococcus aureus in $19.5 \%$. This distribution of bacterial pathogens is similar to what has been reported by Berbari et al [3]. Other studies looking at microorganisms in periprosthetic hip and knee infection also noted $S$ aureus and coagulase negative Staphylococci to be the most frequent $[18,19]$.

Not surprisingly, we found that receipt of antibiotics in the previous 90 days had a significant association with acquisition of fungal PJI. Previous antibiotic use selects for candidal growth and to that extent, biofilm formation over joint prosthesis is a central process of pathogenesis [1, 20-22]. Additionally, large joints such as the hip and knee are at a higher risk for developing infection due to inherently low blood flow to the cortical bone and occasional formation of a hematoma in a large dead space around the prosthetic device. Hematomas can devascularize adjacent tissues and prevent delivery of antibiotics [6].

Our study found that an association between wound drainage lasting more than 5 days and being at risk for fungal PJI. Ongoing wound drainage (especially under broad spectrum antibiotics for prior bacterial infection) allows superinfection with other pathogens (eg, candida here). One could argue that optimizing the wound environment such that we can reduce fungal or bacterial deposition into the wound could potentially decrease the risk of subsequent infection [23].
Co-morbid conditions including diabetes mellitus type II, renal disease, immunosuppression, malignancy and hemodialysis dependence have been reported to be potential risk factors for fungal PJI [10, 24]. In our study, $53.6 \%$ of cases had at least one co-morbid condition. However, this level of comorbidity was observed in similar frequency in the bacterial cohort. This is not surprising, given that these risk factors are also associated with bacterial PJI. None of the patients had HIV or an immunodeficiency associated condition. In one large review study performed in the Netherlands by Kuiper et al [25], 164 cases of fungal PJI were reviewed from 1966 to July 2012 and $68 \%$ of the patients had one or more risk factors for fungal PJI. In another study by Azzam et al [10], half of the patients with fungal PJI had one or more established risk factors. A more recent study by L. Escola- Verge et al, nearly $80 \%$ had an identifiable risk factor for candida infection [30].

As noted in the previously published studies, lab data including CRP and sedimentation rate does not help discriminate fungal from bacterial PJI [26]. However, in our study, we observed that the median CRP and sedimentation rates were more often lower in fungal PJIs compared to bacterial PJIs. Additionally, we observed in the univariable analysis a relatively lower median synovial fluid leukocyte count among fungal cases $(P=.007)$. The lower serum CRP levels as well as synovial fluid counts in fungal PJIs may suggest that the inflammatory response is not as robust as one would expect to see in a bacterial infection.

Referencing back to the study done by Kuiper et al [25], in which after an extensive search of literature, the authors identified 164 cases of fungal PJIs, half had prosthetic loosening described radiographically. This is similar to what has been reported in patients with bacterial PJIs [27]. In contrast, our study noted that only $26.8 \%$ of patients had loosening in the context of fungal PJIs, but this was deemed not to have a statistically significant association.

The hallmark of our study was the relatively large number of cases $(82.9 \%)$ with history of a prior two-stage exchange $(P=.008)$. To our knowledge, this is the first study to establish an association between a patient's surgical history and subsequent development of fungal PJI. In a study done by Geng et al [28], which was a retrospective analysis of eight cases of fungal PJIs, six had undergone additional surgery on the infected joint following primary total joint arthroplasty and preceding diagnosis of fungal PJI. In a second smaller study from Spain, comprising 10 patients with fungal PJIs, nine had previous bacterial infections, received antibiotics for more than 15 days and required multiple surgeries [29]. We infer 
that prior two-stage exchange may increase the risk of fungal infection, possibly mediated through an increased exposure to antibiotics (for previous episode of infection) leading to selection for candida species.

Strengths of this study include a strict definition of cases and controls as well as potential risk factors. We only included cases where we had demonstration of fungi in at least two samples (tissue/fluid). By excluding mixed cultures, we eliminated bias as patients with mixed cultures would be inherently a different population than those infected by a single pathogen. Given the retrospective nature of this study, there might have been some inherent limitations with regards to the recording of data. Also, certain risk factors that occur infrequently may not have been identified. $87 \%$ of the patient population was Caucasian; thus the results of our study can apply primarily to the Caucasian population and we cannot make an inference to the role of other races as risk factors due to the small number. Most of the cases (33 of 41) came from Center A, which could have potentially created selection bias, however we controlled for center in the final model. Both centers acquired and collected data in a similar way and both centers used the same software. As explained by L. Escola- Verge et al, searching and treating for candida intertrigo could be a reasonable measure to avoid candida PJI [30]. We were not able to record how many patients had cutaneous candidiasis as that can be a potential risk factor.

\section{Conclusions}

Our study suggests that receipt of antibiotics within the previous 90 days and wound drainage for more than five days were independent risk factors for fungal PJI. More than half of the patients had at least one risk factor predisposing to fungal PJI and recognition of these risk factors could help clinicians anticipate fungi as the cause of PJI and potentially help to alter medical and surgical treatment strategies.

\section{Abbreviations}

PJI: prosthetic joint infection; THA: total hip arthroplasty; TKA: total knee arthroplasty; AKI: acute kidney injury.

\section{Competing Interests}

The authors have declared that no competing interest exists.

\section{References}

1. Kojic EM, Darouiche RO. Candida infections of medical devices. Clin Microbiol Rev 2004; 17(2): 255-67.

2. Vessely MB, Whaley AL, Harmsen WS, Schleck CD, Berry DJ. The Chitranjan Ranawat Award: Long-term survivorship and failure modes of 1000 cemented condylar total knee arthroplasties. Clin Orthop Relat Res 2006; 452: 28-34.

3. Berbari EF, Hanssen AD, Duffy MC, et al. Risk factors for prosthetic joint infection: case-control study. Clin Infect Dis 1998; 27(5): 1247-54.

4. Zimmerli W, Trampuz A, Ochsner PE. Prosthetic-joint infections. N Engl J Med 2004; 351(16): 1645-54.

5. Sculco TP. The economic impact of infected joint arthroplasty. Orthopedics 1995; 18(9): 871-3.

6. Stocks G, Janssen HF. Infection in patients after implantation of an orthopedic device. ASAIO J 2000; 46(6): S41-6.

7. Kurtz S, Ong K, Lau E, Mowat F, Halpern M. Projections of primary and revision hip and knee arthroplasty in the United States from 2005 to 2030. J Bone Joint Surg Am 2007; 89(4): 780-5.

8. Dale H, Fenstad AM, Hallan G, et al. Increasing risk of prosthetic joint infection after total hip arthroplasty. Acta Orthop 2012; 83(5): 449-58.

9. Tande AJ, Patel R. Prosthetic joint infection. Clin Microbiol Rev 2014; 27(2): 302-45

10. Azzam K, Parvizi J, Jungkind D, et al. Microbiological, clinical, and surgical features of fungal prosthetic joint infections: a multi-institutional experience. J Bone Joint Surg Am 2009; 91 Suppl 6: 142-9.

11. Namba RS, Inacio MC, Paxton EW. Risk factors associated with deep surgical site infections after primary total knee arthroplasty: an analysis of 56,216 knees. J Bone Joint Surg Am 2013; 95(9): 775-82.

12. Klatte TO, Kendoff D, Kamath AF, et al. Single-stage revision for fungal peri-prosthetic joint infection: a single-centre experience. Bone Joint J 2014; 96-B(4): 492-6.

13. Chandra J, Kuhn DM, Mukherjee PK, Hoyer LL, McCormick T, Ghannoum MA. Biofilm formation by the fungal pathogen Candida albicans: development, architecture, and drug resistance. J Bacteriol 2001; 183(18): 5385-94.

14. Aletaha D, Neogi T, Silman AJ, et al. 2010 rheumatoid arthritis classification criteria: an American College of Rheumatology/European League Against Rheumatism collaborative initiative. Ann Rheum Dis 2010; 69(9): 1580-8.

15. Classification and diagnosis of diabetes mellitus and other categories of glucose intolerance. National Diabetes Data Group. Diabetes 1979; 28(12): 1039-57.

16. Clinical Guidelines on the Identification, Evaluation, and Treatment of Overweight and Obesity in Adults--The Evidence Report. National Institutes of Health. Obes Res 1998; 6 Suppl 2: 51S-209S.

17. Kuhn DM, Chandra J, Mukherjee PK, Ghannoum MA. Comparison of biofilms formed by Candida albicans and Candida parapsilosis on bioprosthetic surfaces. Infect Immun 2002; 70(2): 878-88.

18. Giulieri SG, Graber P, Ochsner PE, Zimmerli W. Management of infection associated with total hip arthroplasty according to a treatment algorithm. Infection 2004; 32(4): 222-8.

19. Laffer RR, Graber P, Ochsner PE, Zimmerli W. Outcome of prosthetic knee-associated infection: evaluation of 40 consecutive episodes at a single centre. Clin Microbiol Infect 2006; 12(5): 433-9.

20. Kumamoto CA. Candida biofilms. Curr Opin Microbiol 2002; 5(6): 608-11.

21. Tsui C, Kong EF, Jabra-Rizk MA. Pathogenesis of Candida albicans biofilm. Pathog Dis 2016; 74(4): ftw018.

22. Silva S, Negri M, Henriques M, Oliveira R, Williams DW, Azeredo J. Adherence and biofilm formation of non-Candida albicans Candida species. Trends Microbiol 2011; 19(5): 241-7.

23. Hanssen AD, Osmon DR, Nelson CL. Instructional Course Lectures, The American Academy of Orthopaedic Surgeons - Prevention of Deep Periprosthetic Joint Infection. J Bone Joint Surg 1996; 78(3): 458-71.

24. Anagnostakos K, Kelm J, Schmitt E, Jung J. Fungal periprosthetic hip and knee joint infections clinical experience with a 2-stage treatment protocol. J Arthroplasty 2012; 27(2): 293-8.

25. Kuiper JW, van den Bekerom MP, van der Stappen J, Nolte PA, Colen S. 2-stage revision recommended for treatment of fungal hip and knee prosthetic joint infections. Acta Orthop 2013; 84(6): 517-23.

26. Bracken CD, Berbari EF, Hanssen AD, Mabry TM, Osmon DR, Sierra RJ. Systemic inflammatory markers and aspiration cell count may not differentiate bacterial from fungal prosthetic infections. Clin Orthop Relat Res 2014; 472(11): 3291-4.

27. Bernard L, Lubbeke A, Stern R, et al. Value of preoperative investigations in diagnosing prosthetic joint infection: retrospective cohort study and literature review. Scand J Infect Dis 2004; 36(6-7): 410-6.

28. Geng $\mathrm{L}, \mathrm{Xu} \mathrm{M}, \mathrm{Yu} \mathrm{L}$, et al. Risk factors and the clinical and surgical features of fungal prosthetic joint infections: A retrospective analysis of eight cases. Exp Ther Med 2016; 12(2): 991-9.

29. Garcia-Oltra E, Garcia-Ramiro S, Martinez JC, et al. [Prosthetic joint infection by Candida spp.]. Rev Esp Quimioter 2011; 24(1): 37-41.

30. Escolà-Vergé L, et al. Journal of Infection 77 (2018) 151-157. 
31. Timothy B, Stephen P, Douglas O, et al. Periprosthetic joint infection with fungal pathogens. J Arthroplasty. 2018;33(8):2605-2612. 